\title{
"Laboratory Parameters in Clinically Suspected Dengue Cases in Tertiary Care Teaching Hospital in North Maharashtra"
}

\author{
Madhuri Magan Suryawanshi*, Shubhngi C. Dange, M. N. Dravid, Sunil P. Lilani, Pooja Shah \\ Dept. of Microbiology, Shri Bhausaheb Hire Govt. Medical College Dhule Maharashtra India
}

\section{ABSTRACT}

Background: Dengue an arbovirus infection, has significantly increased in past decade causing increased mortality \& morbidity in temperate countries including India. Earlier diagnosis helps in prompt treatment resulting in decrease in mortality \& prevention of complications. In present study we have evaluated laboratory parameters for diagnosis along with different demographic profile for prevention of disease.

Methods: All clinically suspected patients tested for NS1Ag \& IgM by ELISA along with platelet count \& Peripheral blood smear. Individual results were used in comparative analysis according to demographic (gender, age) and laboratory (platelet counts, NS1 Ag \& IgM) profiles

Result: Total serologically confirmed cases were 583(21.12\%). Lower platelet counts was the most important factors in predicting dengue infection. Furthermore, all demographic and laboratory profiles presented a conservative temporal pattern throughout this long-lasting outbreak.

Conclusion: As consistency throughout the epidemic facilitated defining the conservation pattern throughout the early stages, this was useful for improving management during the remaining period. Also NS1 Ag is early \& reliable test.

Keywords: Demographic Profile, Dengue Fever, NS1 Ag, IgM ELISA

\section{Introduction}

Dengue an important emerging disease of tropical \& sub tropical region is a Vector born disease is caused by Dengue Virus belonging to the family Flaviviridae \& genus Flavivirus. Globally dengue is most important mosquito born viral disease presenting with varied clinical symptoms ranging from flue like fever to deadlier DHF \& Shock. ${ }^{(1)}$.

Epidemiologically, globally 50 million people are infected with dengue virus every year while $2 \&$ half billions are at risk of infection ${ }^{2}$. Similarly in India with population of more than 1 billion, it is estimated that India has largest number of dengue cases, with about 33 millions apparent $\&$ another 100 million asymptomatic infections occurring annually. ${ }^{3}$ The first epidemic of clinical dengue-like illness was recorded in Madras (now Chennai) in 1780 and the first virologically proved epidemic of DF in India occurred in Calcutta and Eastern Coast of India in 1963-1964.4/5/6.

The first major wide spread epidemics of DHF/DSS occurred in India in 1996 involving areas around Delhi ${ }^{7}$ and Lucknow ${ }^{8}$ and then it spread to all over the country ${ }^{9}$ India has documented infections from all four serotypes.

According to WHO, the incidence of dengue globally has shot up 30 folds in last 50 yrs. The cumulative dengue diseases burden has attained an unprecedented proportion in recent times with a sharp increase in the size of population at risk. Dengue disease presents highly complex pathophysiological, economic \& ecological problem ${ }^{10}$

Currently neither effective antiviral agents to treat dengue infection nor an effective vaccine are available. So main approach for prevention, control \& management of dengue lays with vector control \& early diagnosis of disease.

In current study, we have studied different parameters like seasonal variations, gender, age along with laboratory parameters in relation with clinical symptoms which will help us to determine burden of disease in this region \& preventive measures to be taken.

\section{Materials and Methods}

Study was conducted at SBH GMC, Dhule after the Ethical committee approval. Study caters to both urban population and referred rural cases during the period Jan 2016-Dec 2016 .Blood samples were collected in the plain \& EDTA bulb from patients clinically diagnosed as dengue fever .The serum was separated. Cold chain was maintained during transportation of the serum samples whenever required. Data were extracted from patient's notes collect to socio-demographic factors, clinical characteristics of presenting illness, probable diagnosis, management \& outcome of disease. In laboratory investigations Platelet count, Peripheral Blood Smear for Malarial Parasite was done for all cases .Investigations like NS1 antigen ELISA, IgM antibody ELISA were done on all serum samples. 
Cases with Positive NS1 antigen, IgM Dengue Antibody or both were recognized as Dengue fever.( NS1 antigen ELISA(Dengue NS1Ag MICROLISA)\&IgM antibody ELISA kits(NIV DEN IgM Capture ELISA Kit) were standardized and supplied by NIV Pune. Ig G antibody is not reliable marker as it is a cross reacting antibody to other flaviviruses also hence not considered in this study. ${ }^{11}$

\section{Result}

Clinically suspected dengue patients from Jan. 2016 to Dec 2016 were included in study. Total 2755 samples from patients were collected $\&$ tested as per protocol. Patients tested positive for malaria were excluded from study. Of these 583 were confirmed dengue cases \& remaining 2172 were considered as dengue negative $\&$ appropriate control. Among the dengue positive cases,67.93\% ( 396/583) were male \& 32.07\% (187/583) were female. (Table 1)

A substantial number of patients belonged to the age group of 16-30 yrs i.e. 325(55.75\%) followed by age group 1 month -15 yrs. i.e. 156 (26.7\%). Youngest case was diagnosed at age of 4 months with total 13 cases were detected in less than $1 \mathrm{yr}$ age group.

During Study period highest number of cases were noted in the month of September $210(36 \%)$ followed by October 171(29.34\%) \& November 93(15.95) respectively. Sporadic cases were noted throughout year.( Figure 1)

Out of 2755 serum samples tested, 583 (21.16\%)were positive for either one or both serological marker. Of these 583 sample $378(64.8 \%$ ) were exclusively positive for NS1 antigen while 167 ( 28.6\%) sample showed IgM antibody titer positivity. 38 serum samples were positive for both serological marker i.e. NS1 antigen \& IgM antibody (Table 2)

Among 583 sero-positive cases thrombocytopenia (Platelets $<1 \mathrm{Lac}$ ) was evident in 299 (51.29\%) while out of 2172 seronegative cases thrombocytopenia was seen in 159(7.3\%) Out of the $378 \mathrm{NS} 1$ positive cases, 224( 59.26\%) had platelet count less than $100,000 / \mathrm{ml}$ while out of $167 \mathrm{IgM}$ positive samples, 83(49.7\%) had thrombocytopenia. (Table $3 \& 4$ )

Table:1 Age, Sex \& Month wise distribution of cases.

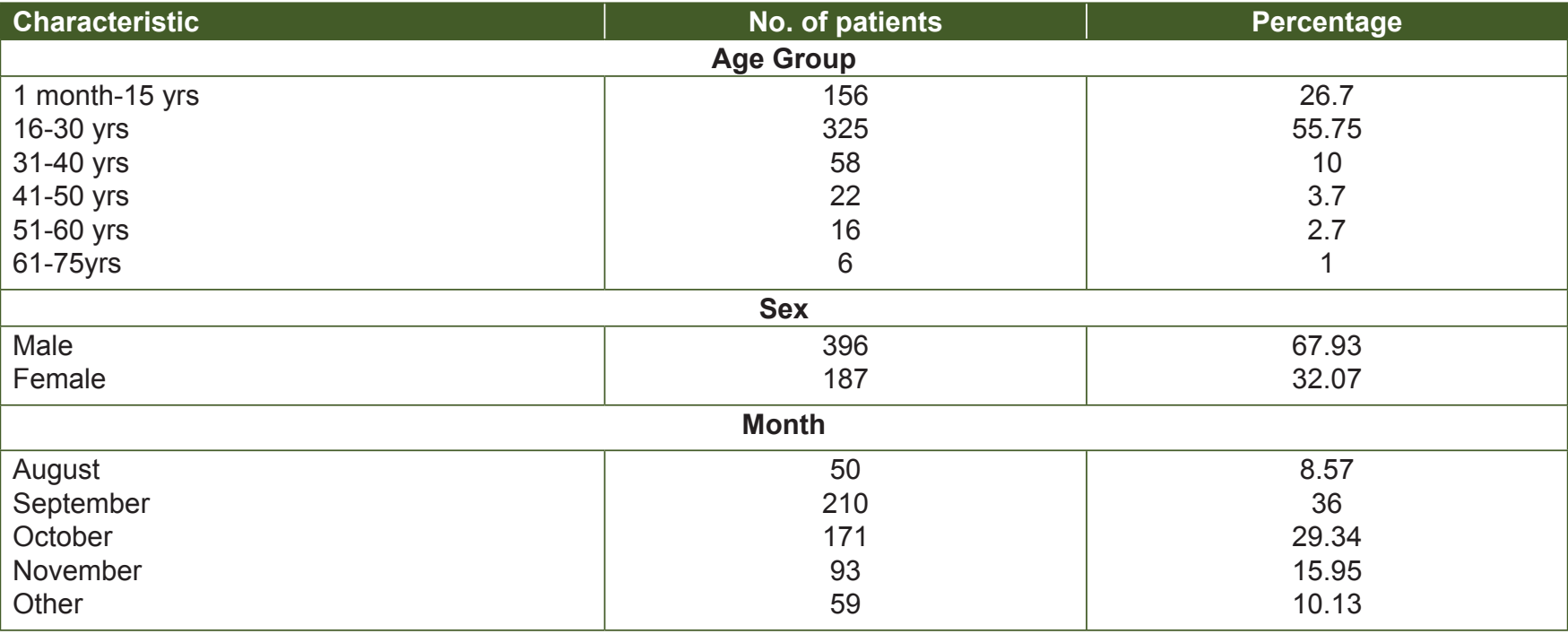

Table 2: Serological marker wise distribution of cases.

\begin{tabular}{|l|c|c|}
\hline Dengue specific marker & Total number of positive serum sample & Percentage \\
\hline NS 1antigen only & 378 & 64.8 \\
\hline IgM Only & 167 & 28.6 \\
\hline NS1+ IgM & 38 & 6.6
\end{tabular}

Table: 3 Comparison of Dengue marker \& Platelet count.

\begin{tabular}{|l|c|c|c|}
\hline Dengue specific marker & Total positive serum sample & Platelet counts (<1,00,000) & Percentage (\%) \\
\hline NS 1 Antigen only & 378 & 224 & 59.26 \\
\hline IgM Only & 1867 & 83 & 49.7 \\
\hline NS1+ IgM & 38 & 12 & 31.5 \\
\hline Total & $\mathbf{5 8 3}$ & $\mathbf{2 9 9}$ & $\mathbf{5 4 . 7}$ \\
\hline
\end{tabular}


Table: 4 Study of dengue cases with platelet count $(n=583)$.

\begin{tabular}{|c|c|c|}
\hline Platelet range & Dengue positive cases & Dengue negative cases \\
\hline Platelet counts less than $<1,00,000 / \mathrm{cmm}$ & 299 & 159 \\
\hline Platelet counts more than $>1,00,000 / \mathrm{cmm}$ & 284 & 2013 \\
\hline Total & 583 & 2172 \\
\hline
\end{tabular}

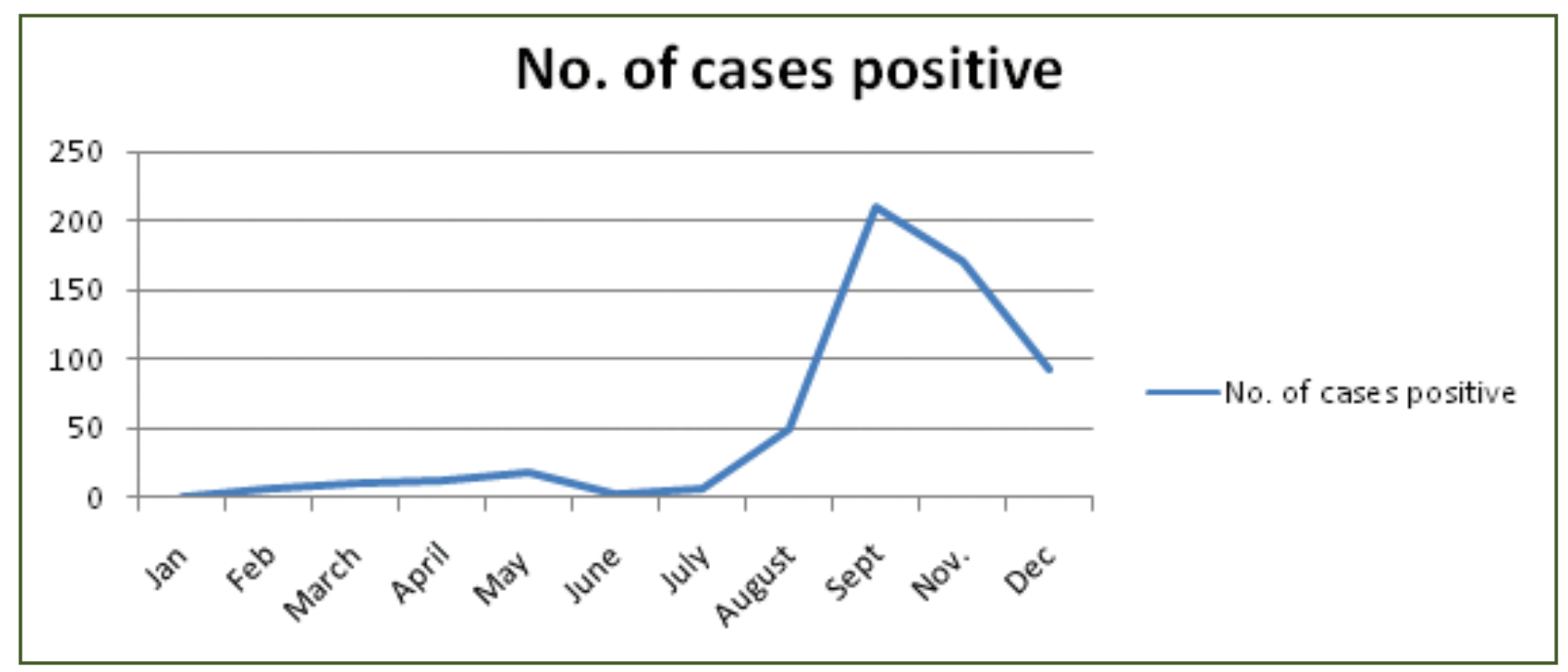

Fig. Line Diagram showing month wise break down of cases.

\section{Discussion}

Dengue is an important emerging disease of the tropical $\&$ sub tropical regions today. India is one of the seven countries in the South East Asia, endemic for DF \& Dengue Hemorrhagic Fever \& may soon become a major niche for dengue infection. ${ }^{12}$ In present study, maximum numbers of patients suffering from dengue were in age group of16-30 yrs. This finding is in accordance with study done by Doke et al in Maharashtra ${ }^{13}$ Mohamed Murtuza Kauser et al ${ }^{14}$ from Central Karnataka, Kumar et al from Udupi Karnataka ${ }^{12}$ Hemant Kumar et al from Surat ${ }^{15}$. Though maximum positive cases belonged to young adult group who are exposed to more outdoor activities, extreme age variations were observed. Youngest patient in present study was 4 months old while oldest was 72 yrs in age. The youngest patient reported by Jonathan G. Lim et al in a study done in 2000-04 was 4 months old ${ }^{16}$. M.J. Kulkarni et al reported 6 cases of newborn admitted for dengue ${ }^{17}$ evidently showing all ages are more or less susceptible for dengue infection.

Further majority of patients were male (396) while female constitute rest (187) thus male to female ratio is 2.1: 1. M.J. Kulkarni in 2010 found two third patients to be males ${ }^{17}$ In contrast to this, a study reported by CV Prathyusa et al from Andhra Pradesh state showed almost equal distribution of male and female ratio in $2012^{18}$. Equal sex distributions was reported by study of Jonathan G. Lin et al from Malayasia ${ }^{16}$. Male to female ratio in a study by Mohan Kashikanti et al in 2013 found a ratio of 1.2:19 Majority of patients $(92.03 \%)$ were found to be males and females constituted $7.96 \%$ by a study done by Hemant Kumar et al ${ }^{15}$ A. Abrol et al reported male to female ratio of 1:1 from a study in Chandigarh ${ }^{20}$

The present study shows that outbreak occurred from August to December. Dengue outbreak in India have generally occurred between August and November ${ }^{21}$ M.J. Kulkarni et al in their study of Dengue cases in a tertiary care center in Jaipur reported cases from September to November ${ }^{17}$ Mohan D.K. reported most of the cases during the month of June to September ${ }^{19}$ A study of 766 patients of Dengue in 2011-2013 in A.J. Institute of Medical Sciences and Research Center, Mangalore, Karnataka, reported cases throughout the year with peak admission in April followed by May and June. ${ }^{15}$

Before the advent of NS1 Ag testing, demonstration of dengue specific $\operatorname{IgM} / \mathrm{Ig} G$ antibodies was mainstay for diagnosis of dengue infection since viral culture or detection of viral genome by molecular method is not feasible at grass root level. As antibodies begin to appear on $5^{\text {th }}$ day of fever in primary $\& 3^{\text {rd }}$ day in secondary infection, diagnosis is delayed due to lag period in this test. In contrast NS1 Ag is detectable in blood from day 1 of fever $\&$ can be seen up to 9 day even when viral RNA detection is negative by RT PCR. ${ }^{22}$ 
Out of total 583 sero-positive cases, 378(64.8\%) were positive for NS1 Ag, 167(28.6\%) for IgM Antibody \& $38(6.6 \%)$ for both. Similar studies by Santosh Tathe et al ${ }^{23}$ shows NS1 Positivity in $88.23 \%$, IgM 68.75\% \& both NS1+ IgM 100\% respectively. Also Sindhanai V et al documented $86.5 \%$ NS 1 positivity with $44.4 . \%$ positivity for IgM. ${ }^{24}$ While most studies shows more NS1 positivity compared to IgM, study by Mohite et al ${ }^{25}$

This study also tried to find the association of thrombocytopenia with dengue parameter. Among 583 seropositive dengue cases thrombocytopenia was detected in $299(51.3 \%)$ while only $159(7.3 \%)$ cases of 2172 were thrombocytopenic, signaling importance of thrombocytopenia as precious clue for diagnosis.

Out of 378 NS1 positive cases, 224(59.26\%) showed thrombocytopenia while $83(49.7 \%)$ of exclusively IgM positive cases were thrombocytopenic. This difference was statistically significant using $\mathrm{Z}$ test. This is in accordance with study by Tanvi Panwala et al ${ }^{26}$, Kulkarni et al ${ }^{17}$. It also signifies importance of doing NS1 Ag detection test in all febrile patients with thrombocytopenia.

\section{Conclusion}

The present study shows that dengue infection is more common in young adult age group favoring males as this group more involved in outdoor activities, which exposes them to mosquitoes. Since monsoon in our region in not heavy \& has intermittent showers, peak of case is seen during mid \& end of monsoon. For all febrile patients with thrombocytopenia, Dengue NS1 Ag, IgM antibodies detection should be done as low platelet is important but not exclusive test for this infectious disease.

\section{Acknowledgements}

We are thankful to Mrs. Suhasini Gavit for her technical support. We also extend thanks to authors /editors/ publishers of all those articles, journals and books from where the literature for this article has been reviewed and discussed.

\section{Reference}

1. WHO Classification of Dengue cases D Gupta-sapir \& B. Schimmer, "Dengue fever: New paradigms for changing epidemiology". Emerging Themes in Epidemiology, Vol 2, article 1, 2005.

2. Bhatt S, Gething PW, Brady OJ, et al. The global distribution \& burden of dengue . Nature 2013; 496: 504-507

3. SarkarJK, Chatterjee SN, Chakravarty SK. Haemorrhagic Fever in Calcutta: some epidemiological observations. Indian J Med Res 1964; 52 : 651-9.

4. Chatterjee SN, Chakravarti SK, Mitra AC, Sarkar JK. Virological investigation of cases with neurological complications during the outbreak of haemorrhagic fever in Calcutta. J Indian Med Assoc 1965; 45 : 314-6.

5. Carey D7. E, Myers RM, Reuben R, Rodrigues FM. Studies on dengue in Vellore, South India. Am J Trop Med Hyg 1966; $15: 580-7$.

6. DarL, Broor S, Sengupta S, Xess I, Seth P. The first major outbreak of dengue hemorrhagic fever in Delhi, India. Emerg Infect Dis 1999; 5 : 589-90.

7. Agarwal R, Kapoor S, Nagar R, Misra A, Tandon R, Mathur A, et al. A clinical study of the patients with dengue hemorrhagic fever during the epidemic of 1996 at Lucknow, India. Southeast Asian J Trop Med Public Health 1999; 30 : 735-40.

8. Shah I, Deshpande GC,TardejaPN. Outbreak of dengue in Mumbai and predictive markers for dengue shock syndrome. J Trop Pediatr2004; $50:$ 301-5.

9. Gubler DJ. The economic burden of dengue . Am. J. Trop. Med. Hyg. 2012; 86; 743-744

10. Shrivatavaa, Dash PK, Tripathi NK, Sahni AK et al" Evaluation of a commercial dengue NS1enzyme linked immune-sorbent assay for early diagnosis of dengue infection. Indian J. Med. Microbiology 2011; 29:51-5)\

11. Kumar A, Rao CR, Pandit V, Shetty S, Bammigatti C, Samarasinghe CM. Clinical Manifestations \& Trend of dengue cases admitted in a Tertiary Care Hospital Udupi District, Karnataka. Indian Journal of Community Medicine: Official Publication of Indian Association of Preventive \& Social Medicine. 2010;35(3):386-90).

12. Prakash Doke, Satish Pawar: Profile of dengue fever outbreak in Maharashtra; Indian Journal of Community Medicine 2000; Vol 25, No 4 (2000-10-2000-12)

13. Mohamed Murtuza Kauser, Kalavathi GP, Mehul Radadiya, Karthik M, Asfiya Afreen, Kumarswamy R. C. : A study of clinical \& LaboratoryProfile of Dengue fever in Tertiary Care Hospital in Central Karnataka, India. Global Journal of Medical Research(B) volume XIV Issue V Version I Yr 2014 Page 7-12

14. Hemant Kumar, Saba Mohmmed Mansoor : A study of Clinico- Demographic profile of Dengue cases in A Teaching Hospital. National Journal of Community Medicine 2015/13557:2038)

15. Jonathan G. Lim, Salvacion R, Gatchalian Ma, Rosario Z. Capeding. Profile of Pediatric patients with DF/ DHF over a Five Year Period( 2000-04). Pediatric Infectious Disease Society of Philippines Journal (2010) 11 (1) 26-34)

16. Manjunath J. Kulkarni, Vijayasarathi, Vikas Bhalla, Deepak Shivpuri, Usha Archarya. Clinico-Epidemiological profile of Children Hospitalized with Dengue. Indian Journal of Pediatr(2010) 77: 1103-1107.

17. C.V.Prathyusha, M.SrinivasaRao, P.Sudarsini and K.UmamaheswaraRao. Clinico-haematological profile and outcome of dengue fever in children. Int.J.Curr. Microbiol. App.Sci 2013; 2(10): 338-346 
18. Mohan D Kashinkunti, Shiddappa, Dhananjaya M. A Study of Clinical Profile of Dengue Fever in a Tertiary Care Teaching Hospital. Sch. J. App. Med. Sci., 2013; 1(4):280-282.

19. A Abrol, ADewan, $\mathrm{N}$ Agarwal, A Galhotra, N Goel, H Swami. A Clinico-Epidemiological Profile of Dengue Fever Cases in a Peri-Urban Area of Chandigarh. The Internet Journal of Epidemiology. 2006 Volume 5 Number 1)

20. V K Singh, J M Haria, S K Jain. Hospital Based Study of Dengue Hemorrhagic Fever in Western Uttar Pradesh Region.International Journal of Scientific Study. (2014) 1 (5): 32-34.

21. Alcon S, Talarmin A, Debruyne M, Falconar A, Deubel V, Flamand M. Enzyme-linked immunosorbent assay specific to dengue virus type 1nonstructural protein NS1 reveals circulation of the antigen in the blood during the acute phase of disease in patients experiencing primary or secondary infections. J Clin Microbiol 2002;40:376-81.

22. Santosh Shivajirao Tathe, Chincholkar VV, Kulkarni DM, Nilekar SL, Ovhal RS \& Halgarkar CS: A study of NS1 antigen \& Platelet count for early diagnosis of dengue infection. Int. J. Curr. Microbiology \& Applied science. Vol 2 Number 12 (2013) PP40-44

23. Sindhanai V, Sageera Bano, Rajkumar N, Suresh Chander $\mathrm{VC}$ : Evaluation of correlation between dengue serological markers \& Platelet count. Scholars journal of applied medical sciences, 2016; $4\{2 \mathrm{D}\}: 618-622$

24. Mohite Ravindra, Karamje C Nisha, Shubhangi A Gadgil, Inamdar Dhanashree, Jadhav Pradnya: Association of IgG, IgM antibodied, N1 antigen \& Platelet count in the diagnosis of Dengue Virus Infection in patients attending Bharati Vidyapeeth Deemed university Medical College \& Hospital , sangali. International Journal of contemporary Medical research. Vol 3, issue 10 oct 2016 page no.2942-43.

25. Tanvi H Panawala, Summaiya A Mulla: Evaluation of two diagnostic methods for dengue virus infection \& its correlation with thrombocytopenia. International Journal of Health \& Allied Sciences Vol 5 Issue 2 Apr-Jun2016 page no88-91.

*Corresponding author:

Dr. Madhuri M. Suryawanshi, 23, Sadguru Colony, Nr. Dollys House Deopur Dhule, Maharashtra India.

Phone: +91 9422755260, 8087482679

Email: dr.madhurijk@gmail.com 